This is an electronic reprint of the original article. This reprint may differ from the original in pagination and typographic detail.

Author(s): Hansen, J.P.; Sorngård, S.A.; Forre, M.; Räsänen, Esa

Title: Quantitative modeling of spin relaxation in quantum dots

Year: $\quad 2012$

Version:

Please cite the original version:

Hansen, J.P., Sorngård, S.A., Forre, M., \& Räsänen, E. (2012). Quantitative modeling of spin relaxation in quantum dots. Physical Review B, 85(35326).

https://doi.org/10.1103/PhysRevB.85.035326

All material supplied via JYX is protected by copyright and other intellectual property rights, and duplication or sale of all or part of any of the repository collections is not permitted, except that material may be duplicated by you for your research use or educational purposes in electronic or print form. You must obtain permission for any other use. Electronic or print copies may not be offered, whether for sale or otherwise to anyone who is not an authorised user. 


\title{
Quantitative modeling of spin relaxation in quantum dots
}

\author{
J. P. Hansen, ${ }^{1,2}$ S. A. Sørngård, ${ }^{1}$ M. Førre, ${ }^{1}$ and E. Räsänen ${ }^{2,3}$ \\ ${ }^{1}$ Department of Physics and Technology, University of Bergen, N-5007 Bergen, Norway \\ ${ }^{2}$ Nanoscience Center, Department of Physics, University of Jyväskylä, P.O. Box 35, FI-40014 Jyväskylä, Finland \\ ${ }^{3}$ Physics Department, Harvard University, Cambridge, Massachusetts 02138, USA
}

(Received 6 January 2011; published 31 January 2012)

\begin{abstract}
We use numerically exact diagonalization to calculate the spin-orbit- and phonon-induced triplet-singlet relaxation rate in a two-electron quantum dot exposed to a tilted magnetic field. Our scheme includes a threedimensional description of the quantum dot, the Rashba and the linear and cubic Dresselhaus spin-orbit coupling, the ellipticity of the quantum dot, and a full angular description of the magnetic field. We are able to find reasonable agreement with the experimental results of Meunier et al. [Phys. Rev. Lett. 98, 126601 (2007)] in terms of the singlet-triplet energy splitting and the spin relaxation rate, respectively. We analyze in detail the effects of the spin-orbit factors, magnetic-field angles, and dimensionality and discuss the origins of the remaining deviations from the experimental data.
\end{abstract}

DOI: 10.1103/PhysRevB.85.035326

PACS number(s): 68.65.Hb, 85.35.Be, 71.38.-k

\section{INTRODUCTION}

Quantum dots (QDs) are well-known examples of confined and quantized systems in semiconductor heterostructures. Among other potential applications in, e.g., quantum optics, QDs have been experimentally realized as controllable quantum bits. ${ }^{1}$ On the road to quantum computing applications, it is essential to understand the electronic and spin-dependent relaxation processes in QDs. ${ }^{2,3}$

The coupling between quantized states and lattice vibrations in semiconductor structures has been examined already for decades. ${ }^{4-6}$ In QDs the spin relaxation has attracted attention both theoretically ${ }^{7-14}$ and experimentally. ${ }^{15-20}$ Recently, Meunier et al. ${ }^{15}$ measured the triplet-singlet relaxation rate in a two-electron (quasi-)two-dimensional (2D) QD. They also derived a semiempirical model reproducing the general trends of the experimental data. However, a good agreement was found using a spin-orbit (SO) coupling that depended on the magnetic field and that was significantly smaller than the SO strength reported in earlier studies. ${ }^{21}$

In this work we describe a two-electron QD using both 2D and three-dimensional (3D) models and include the description of (i) a tilted external magnetic field, (ii) the ellipticity of the QD, and (iii) both the Rashba and the linear and cubic Dresselhaus spin-orbit interaction. We point out that none of the previous theoretical approaches have aimed at such a complete description, which is shown to be important when comparing with experiment at the most detailed level. The two-electron states are calculated numerically exactly by diagonalizing the Hamiltonian, and the phonon-induced relaxation rate is obtained from Fermi's golden rule. We find a reasonable agreement with the experimental results, ${ }^{15}$ provided that the $3 \mathrm{D}$ description and a moderate ellipticity of the QD are applied. The remaining discrepancy between experiment and theory might be caused by a more complex dot geometry of the experiment and/or might indicate the limit of the widely used effectivemass approximation for electrons confined by a harmonic potential.

\section{MODEL AND METHOD}

Our two-electron QD exposed to an external magnetic field B is described by the Hamiltonian

$$
H=\sum_{i=1,2}\left[h\left(\mathbf{r}_{i}\right)+h_{\mathrm{SO}}\left(\mathbf{r}_{i}\right)\right]+\frac{e^{2}}{4 \pi \epsilon_{r} \epsilon_{0}\left|\mathbf{r}_{1}-\mathbf{r}_{2}\right|},
$$

where $\mathbf{r}_{i}=\left(x_{i}, y_{i}, z_{i}\right)$ with $i=1,2$ are the coordinates of the two electrons. The single-electron Hamiltonian is given by a sum of the kinetic, external potential, and Zeeman terms as

$$
\begin{aligned}
h= & -\frac{\hbar^{2}}{2 m^{*}}(\nabla+e \mathbf{A})^{2}+\frac{1}{2} m^{*}\left[\delta \omega_{0} x^{2}+(1 / \delta) \omega_{0} y^{2}+\omega_{z} z^{2}\right] \\
& +g^{*} \mu_{B} \mathbf{B} \cdot \mathbf{S} .
\end{aligned}
$$

We consider magnetic fields tilted from the $x y$ plane with an angle $\theta$ and tilted azimuthally from the $y$ axis with an angle $\phi$. Thus the magnetic field has the expression

$$
\mathbf{B}=B_{0}(\cos \phi \sin \theta, \sin \phi \sin \theta, \cos \theta) .
$$

The vector potential in the symmetric gauge, $\mathbf{A}=(\mathbf{B} \times \mathbf{r}) / 2$, then becomes

$$
\begin{aligned}
\mathbf{A}= & \frac{B_{0}}{2}(z \sin \theta \sin \phi-y \cos \theta, x \cos \theta \\
& -z \cos \phi \sin \theta, y \cos \phi \sin \theta-x \sin \phi \sin \theta),
\end{aligned}
$$

which approaches $\mathbf{A}=B_{0}(-y, x) / 2$ in the $2 \mathrm{D}$ limit $(z \rightarrow 0)$.

The harmonic confinement potential is asymmetric both laterally and vertically: the ellipticity in the $x y$ plane can be tuned with the $\delta$ parameter, and the in-plane and offplane confinement strengths are fixed to $\hbar \omega_{0}=2.5 \mathrm{meV}$ and $\hbar \omega_{z}=11.9 \mathrm{meV}$, respectively. The material parameters are chosen to be those of GaAs, i.e., effective mass $m^{*}=0.067 m_{e}$, relative permittivity $\epsilon_{r}=12.4$, and the gyromagnetic ratio $g^{*}=-0.44$. The constant $\mu_{B}=e \hbar /\left(2 m_{e}\right)$ in Eq. (3) is the Bohr magneton.

A harmonic form has been shown to describe well the electron confinement in both lateral and vertical semiconductor (GaAs-type) quantum dots. ${ }^{22}$ The model was established 
soon after the first Coulomb-blockade experiments, when the measured and calculated addition energies were compared. ${ }^{23}$ More recently, the harmonic model has been shown to explicitly yield the measured single-electron spectrum ${ }^{24}$ and also the spin-blockade oscillations for both lateral and vertical QD devices up to about 50 electrons. ${ }^{25,26}$

The SO interaction in Eq. (1) is given by

$$
h_{\mathrm{SO}}=h_{R}+h_{D}
$$

with the Rashba term

$$
h_{R}=\frac{\alpha}{\hbar}\left(p_{x} \sigma^{y}-p_{y} \sigma^{x}\right)
$$

and the Dresselhaus term, ${ }^{27}$ containing both linear and cubic contributions,

$$
h_{D}=\frac{\gamma}{\hbar^{3}}\left\langle p_{z}^{2}\right\rangle\left(p_{y} \sigma^{y}-p_{x} \sigma^{x}\right)+\frac{\gamma}{2 \hbar^{3}}\left(p_{x} p_{y}^{2} \sigma^{x}-p_{y} p_{x}^{2} \sigma^{y}\right) .
$$

The coupling strengths of the Rashba and Dresselhaus terms are set by the parameters $\alpha$ and $\gamma$, respectively, and $\sigma^{x}$ and $\sigma^{y}$ are the Pauli matrices. We choose $\alpha=0$ and $\gamma=11.15 \mathrm{eV} \AA^{3}$ as our default values, but consider also different magnitudes to assess the sensitivity of the spin relaxation to the SO coupling. The measurements of Meunier et al. ${ }^{15}$ and 2D calculations ${ }^{8,28}$ suggest relatively small coupling constants. This is in agreement with the experiment of Zumbühl et al. ${ }^{29}$ reporting $\gamma=9 \mathrm{eV} \AA^{3}$, which is notably smaller than the commonly used value of $27.5 \mathrm{eV} \AA^{3}$.

The total wave function is a product of the $x y$ (planar) component $\psi_{\|}\left(\mathbf{r}_{1}, \mathbf{r}_{2}\right)$, obtained from exact diagonalization of the Hamiltonian in Eq. (1) in a (large) basis of spinsymmetrized products of Cartesian single-electron harmonicoscillator eigenstates, ${ }^{30,31}$ and a simple $z$ (perpendicular) component $\psi_{z}\left(z_{1}, z_{2}\right)$ incorporating the material thickness. The $z$ component of the wave function is conveniently modeled in terms of the ground-state wave function of a harmonic potential, with confinement strength $\omega_{z}$ defining an effective confinement length $\mathcal{L}_{z}=\sqrt{4 \hbar /\left(m^{*} \omega_{z}\right)}$, in the $z$ direction.

The spectrum is obtained by diagonalizing the Hamiltonian in a basis of spin-symmetrized two-electron harmonicoscillator states. Basis sizes $\left|n_{x}, n_{y}, n_{z}\right\rangle$ up to $n_{\max }=6$ for the $(x, y)$ coordinates and $n_{z}=0$ for the vertical coordinate are included. Analytical matrix elements are then obtained for all terms except for the electron-electron interaction in Eq. (1).

It was shown by Popsueva et al..$^{30}$ that, in the $2 \mathrm{D}$ case with $\omega_{x}=\omega_{y}=\omega_{0}$, all matrix elements can be evaluated analytically. In particular, for any double pairs of basis functions $(i, j)$ the four-dimensional integrals over the electron-electron interaction can be expressed as

$$
I_{2 \mathrm{D}}^{r_{12}}=\omega_{0}^{1 / 2} \int_{-\infty}^{\infty} F_{i j}^{2 \mathrm{D}}\left(s_{\|}\right) d s_{\|},
$$

where

$$
F_{i j}^{2 \mathrm{D}}\left(s_{\|}\right)=\sum_{n} a_{n} s_{\|}^{2 n} e^{-s_{\|}^{2}} .
$$

The variable $s_{\|}$is here defined from the Bethe integral,

$$
\frac{1}{\left|\mathbf{r}_{1}-\mathbf{r}_{2}\right|}=\frac{1}{2 \pi^{2}} \int \frac{d^{3} s}{s^{2}} e^{i \mathbf{s} \cdot \mathbf{r}_{1}} e^{-i \mathbf{s} \cdot \mathbf{r}_{2}}
$$

When this expression is introduced in calculating the matrix element between any pair of basis functions $(i, j)$, the expression above is derived with $s_{\|}=\sqrt{s_{x}^{2}+s_{y}^{2}}$. When the integrals have been analytically evaluated once, the matrix elements for any confinement $\omega_{0}$ are obtained by a simple multiplication.

In $3 \mathrm{D}$ the electron-electron repulsion amounts to more complicated six-dimensional integrals. When the calculation is extended with harmonic confinement also in the $z$ direction, a similar scaling relation can be obtained, i.e.,

$$
\begin{aligned}
I_{3 \mathrm{D}}^{r_{12}}= & \omega_{0}^{1 / 2} \int_{-\infty}^{\infty} F_{i j}^{2 \mathrm{D}}\left(s_{\|}\right)\left[1-\operatorname{erf}\left(\sqrt{\frac{1}{2 \omega_{z}}} s_{\|}\right)\right] \\
& \times e^{s_{\|}^{2} / 2 \omega_{z}} d s_{\|} .
\end{aligned}
$$

Here $\operatorname{erf}(x)=2 / \sqrt{\pi} \int_{0}^{x} \exp \left(-t^{2}\right) d t$ is the error function. The matrix elements for the electron-electron interaction can thus be obtained for any $\omega_{0}$ by scaling as in $2 \mathrm{D}$ when they have been calculated for a given value of the vertical confinement. We note also that the formula above provides a continuous route from $3 \mathrm{D}$ to $2 \mathrm{D}$, since

$$
I_{2 \mathrm{D}}^{r_{12}}=\lim _{\omega_{z} \rightarrow \infty} I_{3 \mathrm{D}}^{r_{12}}\left(\omega_{z}\right)
$$

For 2D calculations the finite thickness can be consistently taken into account by replacing Eq. (8) by Eq. (11) when calculating the interaction terms.

The triplet-singlet relaxation rate is calculated using Fermi's golden rule, which-in the case of phonon emissionmay be written as ${ }^{32}$

$\Gamma=\frac{V}{\hbar(2 \pi)^{2}} \sum_{j=1}^{3} \int d^{3} q\left|M_{j}(\mathbf{q})\right|^{2}\left|\left\langle S\left|\widehat{H}_{\mathrm{ph}}\right| T\right\rangle\right|^{2} \delta\left(\Delta E-\hbar c_{j} q\right)$,

where $T$ and $S$ refer to the initial singlet and final triplet states, respectively, $V$ is the normalization volume, $\Delta E$ is the singlettriplet energy splitting, $\mathbf{q}=\left(\mathbf{q}_{\|}, q_{z}\right)$ is the momentum of the released phonon, and $\widehat{H}_{\mathrm{ph}}=\sum_{i=1,2} e^{-i\left(\mathbf{q}_{\|} \cdot \mathbf{r}_{i}+q_{z} z_{i}\right)}$ is the phonon coupling. Linear dispersion relations have been adopted, i.e., we set $\omega_{S, T} \equiv \Delta E / \hbar=c_{l, t} q$. Here $c_{l}=4720 \mathrm{~m} / \mathrm{s} \quad\left(c_{t}=\right.$ $3340 \mathrm{~m} / \mathrm{s}$ ) denotes the longitudinal (transverse) speed of sound. ${ }^{15}$ The electrons couple to longitudinal acoustic phonons through the deformation potential coupling ${ }^{33,34}$

$$
\left|M_{1}(\mathbf{q})\right|^{2}=\frac{\hbar \Xi_{d}^{2}}{2 \rho c_{l} V}|\mathbf{q}|
$$

as well as the piezoelectric coupling

$$
\left|M_{2}(\mathbf{q})\right|^{2}=\frac{32 \pi^{2} \hbar e^{2} h_{14}^{2}}{\epsilon_{r}^{2} \rho c_{l} V} \frac{\left(3 q_{x} q_{y} q_{z}\right)^{2}}{|\mathbf{q}|^{7}},
$$

whereas coupling to transverse phonons takes place only through the latter,

$$
\begin{aligned}
& \left|M_{3}(\mathbf{q})\right|^{2} \\
& \quad=\frac{32 \pi^{2} \hbar e^{2} h_{14}^{2}}{\epsilon_{r}^{2} \rho c_{t} V}\left(\frac{q_{x}^{2} q_{y}^{2}+q_{y}^{2} q_{z}^{2}+q_{z}^{2} q_{x}^{2}}{|\mathbf{q}|^{5}}-\frac{\left(3 q_{x} q_{y} q_{z}\right)^{2}}{|\mathbf{q}|^{7}}\right) .
\end{aligned}
$$


In these expressions $\rho=5300 \mathrm{~kg} / \mathrm{m}^{3}$ is the GaAs mass density, $\Xi_{d}=6.7 \mathrm{eV}$ is the deformation potential constant, and $h_{14}=1.4 \times 10^{9} \mathrm{~V} / \mathrm{m}$ is the piezoelectric constant. There are two transverse phonon modes, and hence $M_{3}(\mathbf{q})$ is considered twice when computing the rate.

The matrix element $\left\langle S\left|\widehat{H}_{\mathrm{ph}}\right| T\right\rangle$ in Eq. (13) separates into a product of a planar $(x y)$ and a perpendicular $(z)$ component, i.e.,

$$
\begin{aligned}
& \left\langle S\left|\widehat{H}_{\mathrm{ph}}\right| T\right\rangle=\sum_{i=1,2}\left\langle\psi_{\|}^{(S)}\left|e^{-i \mathbf{q}_{\|} \cdot \mathbf{r}_{i}}\right| \psi_{\|}^{(T)}\right\rangle\left\langle\psi_{z}\left|e^{-i q_{z} z_{i}}\right| \psi_{z}\right\rangle \\
& =2 \sum_{k, l, k^{\prime}} a_{k, l}^{*} a_{k^{\prime}, l} \Phi_{n_{k}, n_{k^{\prime}}} \Phi_{m_{k}, m_{k^{\prime}}} \exp \left(-\frac{\hbar q_{z}^{2}}{4 m^{*} \omega_{z}}\right) \text {. }
\end{aligned}
$$

Here $\left|\psi_{\|}^{(S)}\right\rangle$ and $\left|\psi_{\|}^{(T)}\right\rangle$ are the planar two-electron singlet and triplet states, respectively. We have assumed that the $z$ component of the wave function is frozen, i.e., no excitations are considered in that direction. The expansion coefficients $a_{k, l}$ are obtained from the diagonalization of the Hamiltonian in Eq. (1). $n_{k}$ and $m_{k}$ are the single-particle harmonic quantum numbers of one of the electrons in the $x$ and $y$ directions, respectively. The factor of 2 in Eq. (17) arises from the fact that the total wave function of each state is antisymmetric. The single-particle matrix elements $\Phi_{n_{i}, n_{i^{\prime}}}=\left\langle\phi_{n_{i}}\left|e^{-i q_{x} x}\right| \phi_{n_{i^{\prime}}}\right\rangle$ are essentially associated Laguerre polynomials in $q^{2}$ multiplied by a damping exponential function,

$$
\Phi_{n, n^{\prime}}=(-i)^{\eta} \frac{N !}{\sqrt{n ! n^{\prime} !}} \tilde{q}_{x}^{2 M+\eta} \exp \left(-\tilde{q}_{x}^{2} / 2\right) L_{N}^{2 M+\eta}\left(\tilde{q}_{x}^{2}\right) .
$$

Here $\tilde{q}_{x}=\hbar q_{x} /\left(2 m^{*} \omega_{0}\right)^{1 / 2}, N=\min \left(n, n^{\prime}\right), M=\left(\left|n-n^{\prime}\right|-\right.$ $\eta) / 2$, and $\eta=1$ if $n+n^{\prime}$ is odd and 0 otherwise (analogously in $y) .{ }^{35}$ The phonon-induced triplet-singlet relaxation rate is now calculated by inserting Eqs. (14)-(17) into Eq. (13) and performing the integration over the phonon momentum q.

\section{RESULTS}

\section{A. Singlet-triplet energy splitting}

Figure 1(a) shows the total energies of singlet (thick lines) and triplet (thin lines) states in a two-electron, elliptic QD as a function of the magnetic field. The ellipticity of the QD is fixed to $\delta=1.3$ [see Eq. (3)]. The triplet states with $S_{z}=0, \pm 1$ are split into three curves due to the Zeeman effect, which is linear in $B$. We show results for three tilting angles of the magnetic field, $\theta=65^{\circ}$ (outermost lines), $50^{\circ}$ (middle lines), and $35^{\circ}$ (innermost lines) as indicated in the figure.

The tilting angle $\theta$ has a relatively large effect on the energies, so that the closing point of the singlet-triplet energy gap $\Delta E$ moves to larger magnetic fields when the angle is increased. Figure 1(b) shows the gap as a function of $B$ in comparison with the experimental values (circles). First, the downward "bending" of $\Delta E$ at small fields is due to the ellipticity; in a circular QD the gap increases linearly as $B$ approaches zero. In this respect, we find a good agreement with the experiment, and may conclude that the ellipticity of the real QD device is close to our chosen value $\delta=1.3$. Second, the best overall agreement is found with a tilting angle
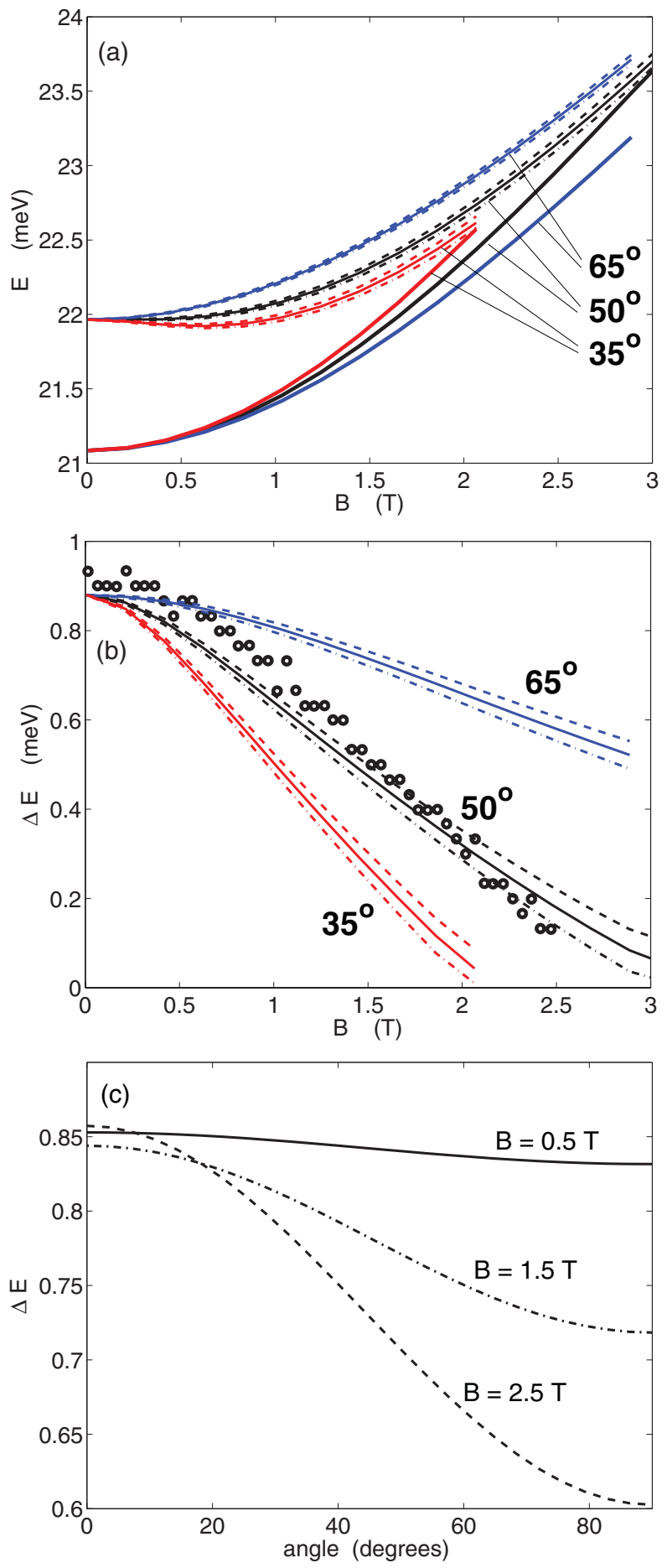

FIG. 1. (Color online) (a) Energy of singlet ground state (thick lines) and three lowest triplet states (thin lines) in a two-electron harmonic $\left(\hbar \omega_{0}=2.5 \mathrm{meV}, \hbar \omega_{z}=11.9 \mathrm{meV}\right)$, elliptic $(\delta=1.3)$ quantum dot as a function of the magnetic-field strength for three different tilting angles of the magnetic field, $\theta=65^{\circ}, 50^{\circ}$, and $35^{\circ}$. In the triplet states the dashed, solid, and dash-dotted lines indicate $S_{z}=-1,0$, and +1 , respectively. (b) Singlet-triplet energy splitting for the three cases of (a). Open circles correspond to the experimental data in Ref. 15. (c) Variation of $\Delta E(B)$ as a function of $\phi$ for $B=0.5 \mathrm{~T}$ (solid line), $1.5 \mathrm{~T}$ (dash-dotted line), and $2.5 \mathrm{~T}$ (dashed line), respectively. The results for $B=1.5 \mathrm{~T}$ and $2.5 \mathrm{~T}$ have been multiplied by factors of 1.4 and 2.4 , respectively, to set the scale. 
$\theta=50^{\circ}$, which is smaller than $\theta_{\text {expt }}=68^{\circ} \pm 5^{\circ}$ reported in Ref. 15.

Another angle affecting the energy gap is the azimuthal direction of the magnetic field with respect to the axis of the ellipticity. In Fig. 1(c) we plot the variation of $\Delta E(B)$ as a function of the azimuthal angle $\phi$ for three magneticfield strengths. We observe that the variation increases with increasing magnetic field, so that close to the crossing $(B \sim 3 \mathrm{~T})$ the variation is $\sim 20 \%$. Combination of this observation with Fig. 1(b) leads to the conclusion that the best agreement with the experimental result is obtained with $\phi=90^{\circ}$, i.e., when the shortest axis of the ellipse is parallel to the tilting direction of the magnetic field. In the following we set $\phi=90^{\circ}$ unless stated otherwise.

With a fixed $\delta$ obtained from the bending as $B \rightarrow 0$ [see Fig. 1(b)], the singlet-triplet energy gap essentially depends on $\omega_{0}$ and $\omega_{z}$. The SO parameters are so small that they play only a minor role in the energy gap. The parameter values used here, $\hbar \omega_{0}=2.5 \mathrm{meV}$ and $\hbar \omega_{z}=11.9 \mathrm{meV}$, give a reasonable agreement with the experiment in the energy separation and in the spin relaxation rate (see below). An increase in both parameters and thus smaller vertical extension can also lead to good agreement in the energy gap, but a poor agreement in the spin relaxation rate. This effect is further illuminated in Sec. III C.

We point out that the results are not sensitive to use of other types of frozen ground-state vertical basis functions and/or a larger number of vertical basis functions. The latter, as well as representing the vertical dimension by an infinite barrier at $z=0$ and a harmonic confinement for $z>0$, does not lead to significant changes. We conclude that good agreement with the experiment is obtained for a narrow range of harmonic confinement parameters. However, the theoretical magnetic tilting angle needs to be reduced to $\theta=50^{\circ}$ to arrive at this level of agreement. The origin of this discrepancy poses a challenge for future experiments and theory. In the remaining part of this work we will stick to $\theta=50^{\circ}$.

\section{B. Spin relaxation rate}

In Fig. 2 we show the calculated total relaxation rate (solid line) in comparison with the experimental result (circles with error bars) in Ref. 15. Dashed and dash-dotted lines correspond to the contributions from the piezoelectric coupling and the deformation potential coupling, respectively. We have used $\gamma=11.15 \mathrm{eV} \AA^{3}$ and $\alpha=0$ for the Dresselhaus and Rashba SO coupling strengths, respectively. Apart from the smallest and largest energy gaps, where the relaxation rate is very small, we obtain a very good agreement with the experimental measurements. We find that interchanging the values between the two couplings has only a minor effect on the transition rate; in fact, this is true for any combination with $\sqrt{\alpha^{2}+\left(\gamma\left\langle p_{z}^{2}\right\rangle\right)^{2}}=0.58 \mathrm{meV} \AA$. The experiment of Meunier et al. ${ }^{15}$ gives a measure of this quantity but cannot resolve the relative magnitude of the two terms. Measurements of angular-resolved phonons ${ }^{36}$ or external fields controlling the Rashba term are needed to address the comparison between theory and experiment at a more detailed level.

In general, the coupling to the phonon bath is strongest when the QD size matches the phonon wavelength., $, 7,15,32$

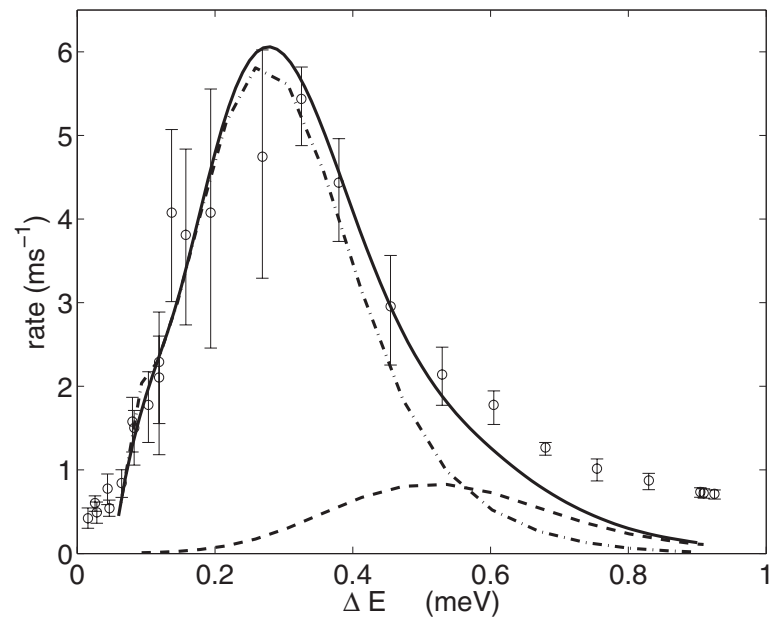

FIG. 2. Calculated total triplet-singlet relaxation rate when the magnetic-field tilting angle is $\theta=50^{\circ}$ (solid line) in comparison with the experimental data in Ref. 15 (circles with error bars). The other parameters are the same as in Fig. 1. The solid line shows the total rate. The dashed and dash-dotted lines show the contributions from the piezoelectric and deformation potential couplings, respectively. The spin-orbit coupling parameters are $\gamma=11.15 \mathrm{eV}^{3}$ and $\alpha=0$ for the Dresselhaus and Rashba terms, respectively.

The piezoelectric coupling (dashed line) is found to dominate the relaxation rate in Fig. 2 at small energy splittings ( $\Delta E \lesssim 0.5 \mathrm{meV}$ ), whereas the deformation potential coupling dominates at larger $\Delta E$. This is expected due to the $\sqrt{\Delta E}$ and $\sqrt{1 / \Delta E}$ dependence of these couplings, respectively. In the former case, the coupling occurs through slower transverse phonons, which yields a peak at smaller $\Delta E$.

Next we examine the effect of the SO coupling strength on the relaxation rate in more detail. Figure 3 shows the scaled relaxation rates for four different sets of $\alpha$ and $\gamma$ in units of $\mathrm{meV} \AA$ and $\mathrm{eV} \AA^{3}$, respectively. The black solid line corresponds to Fig. 2. The other curves are divided by the actual $\alpha^{2}+\left(\gamma\left\langle p_{z}^{2}\right\rangle\right)^{2}$ and multiplied by the square of

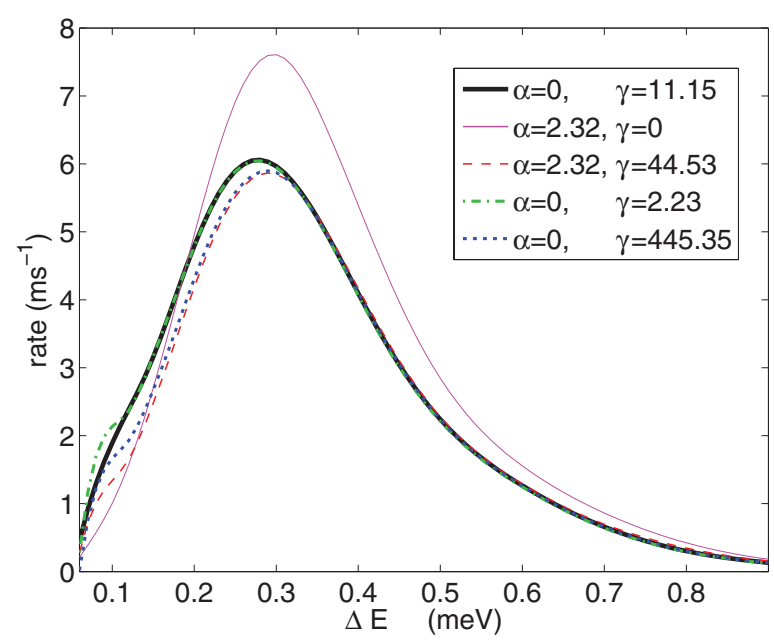

FIG. 3. (Color online) Scaled relaxation rates according to Eq. (19) for four different sets of spin-orbit coupling strengths. $\alpha$ and $\gamma$ are in units of $\mathrm{meV} \AA$ and $\mathrm{eV} \AA^{3}$, respectively. 
the reference value, $\gamma=11.15 \mathrm{eV} \AA^{3}$. Overall, no significant qualitative changes in the rate are obtained within a realistic parameter range. In particular, the peak position is insensitive to both $\gamma$ and $\alpha$, and thus we cannot achieve a better agreement with the experimental rate in Fig. 2 (circles) by tuning either the Dresselhaus or Rashba SO coupling, or both. Furthermore, the fact that all curves are very close to each other indicates that the transition rate scales to a good agreement as

$$
\Gamma(\alpha, \gamma) \approx \frac{\left[\alpha^{2}+\left(\gamma\left\langle p_{z}^{2}\right\rangle\right)^{2}\right]}{\alpha_{0}^{2}+\left(\gamma_{0}\left\langle p_{z}^{2}\right\rangle\right)^{2}} \Gamma\left(\alpha_{0}, \gamma_{0}\right) .
$$

The scaling formula can be derived from an interaction Hamiltonian $V_{I} \sim \alpha V_{I}^{1}+\gamma V_{I}^{2}$ in the first-order perturbation limit when the interfering paths can be neglected, i.e.,

$$
\left\langle f\left|V_{I}^{1}\right| i\right\rangle\left\langle f\left|V_{I}^{2}\right| i\right\rangle \approx 0 .
$$

From Fig. 3 we see that the scaling for fixed $\theta, \phi$ is valid. This suggests that the spin relaxation through the Rashba or Dresselhaus coupling in fact follows noninterfering paths within our basis states.

Generally, however, the SO coupling is anisotropic and depends on the orientation of the magnetic field with respect to the crystal axis. ${ }^{2}$ Consequently, the relaxation rate is anisotropic as well. ${ }^{37}$ Hence, in view of the tilted magnetic field it is difficult to precisely assort the Rashba and Dresselhaus contributions in our QD device.

In Fig. 4 we show how the relaxation rate for fixed magneticfield strength (here $B=2 \mathrm{~T}$ ) depends on the field direction with any combination of $\theta$ and $\phi$. As pointed out above, the relaxation rate is strongly dependent on the energy splitting and qualitatively follows the form $\Delta E^{n} \exp \left(-c \Delta E^{2}\right)$ with positive constants $n$ and $c$. At $B=2 \mathrm{~T}$ the rate is small for both $\theta \sim 0$ and $\theta \sim 90^{\circ}$, corresponding to small- $\Delta E$ and large- $\Delta E$ limits in the qualitative formula, respectively. At intermediate values of $\theta$ we find an area of higher relaxation rates that increases as a function of $\phi$. In this region of increased rates the energy splitting gives phonon wavelengths comparable with the QD size. Detailed analysis of the angular dependence is not the

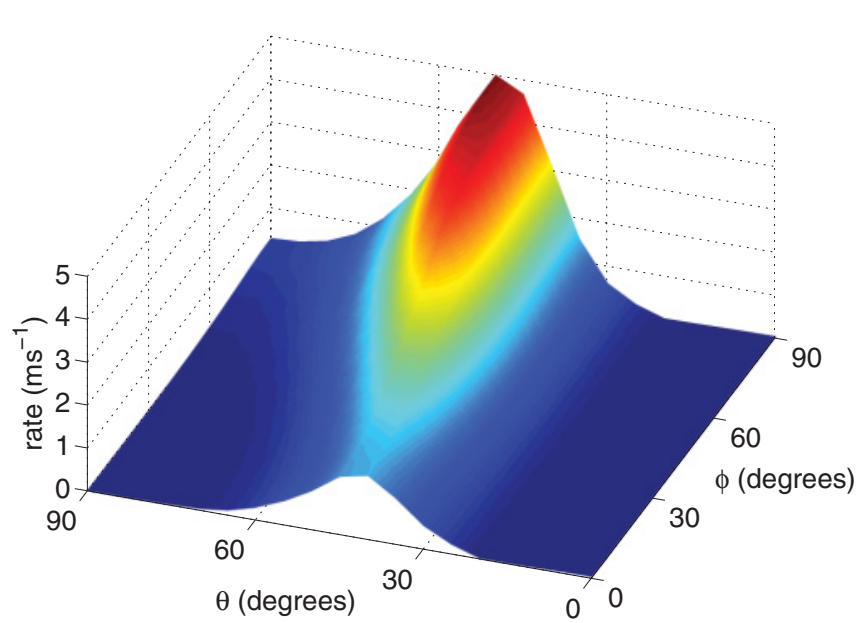

FIG. 4. (Color online) Dependence of the relaxation rate on the magnetic-field angles $\theta$ and $\phi$. The field strength is fixed to $B=2 \mathrm{~T}$. The other parameters are the same as in Fig. 1.
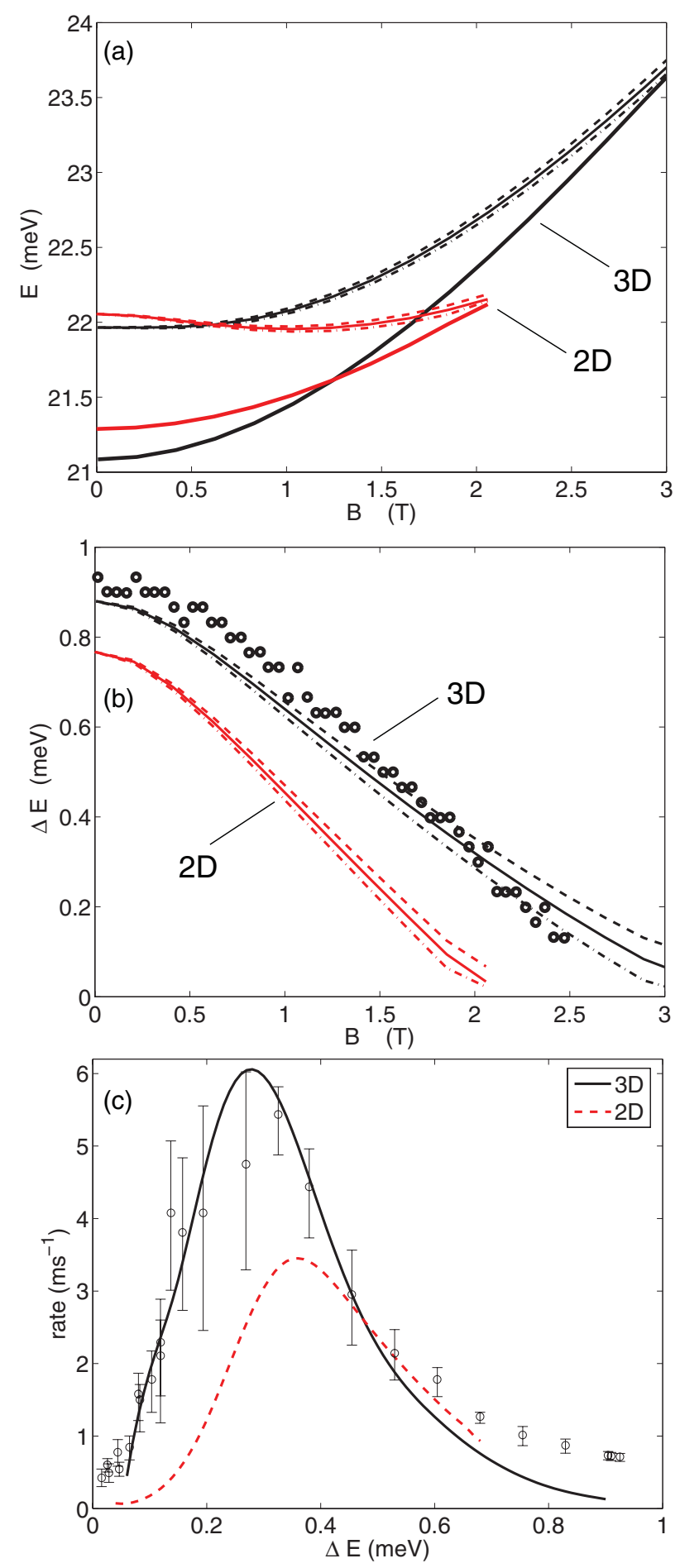

FIG. 5. (Color online) Comparison between two-dimensional and three-dimensional calculations, in both cases with a tilted magnetic field of $\theta=50^{\circ}$. (a) Energy levels of the lowest singlet and triplet states. (b) Singlet-triplet energy splitting. (c) Relaxation rate. Parameters are the same as in Fig. 2.

scope of this work, but we point out that these results could be used as a guideline in the analysis of forthcoming experiments where the direction of the magnetic field can be varied; in principle the angular properties could be used indirectly to obtain precise structural information about the QD device. 


\section{Effects of dimensionality}

Finally we turn our attention to the effects of the dimensionality on the singlet-triplet energy splitting and on the spin relaxation rate. We compare our 3D scheme as described in the beginning of Sec. II to a bare 2D approach, where only the in-plane confinement and 2D basis functions are applied and the 2D approximation for the electron-electron interaction is used (corresponding to $\omega_{z} \gg \omega_{0}$ ). This leads to relatively stronger electronic repulsion in the ground state than in the excited states, which in turn yields a smaller energy splitting.

The results of the comparison are summarized in Fig. 5. First we notice that the tilted magnetic field has a very different effect on the energy states in 3D and 2D [Fig. 5(a)]. In 2D the applied magnetic field closes the energy gap already at $B \sim$ $2 \mathrm{~T}$, whereas in $3 \mathrm{D}$ the closing occurs at $B \sim 3 \mathrm{~T}$. Therefore the $2 \mathrm{D}$ model cannot yield a reasonable agreement with the experimental splitting, as shown in Fig. 5(b). It should be noted that if the confinement strength $\omega_{0}$ in the 2D model is slightly increased to yield the correct splitting at $B=0$, the magneticfield dependence is still wrong, and the rate becomes much worse than the $3 \mathrm{D}$ result in comparison with the experiment. Furthermore, as shown in Fig. 5(c) the 2D model is unable to reproduce the experimental spin relaxation rate. In contrast, the 3D model has a very good agreement with the experiment, as shown above in Fig. 2.

\section{SUMMARY AND DISCUSSION}

To summarize, we have proceeded toward quantitative modeling of spin relaxation in semiconductor quantum dots containing two electrons. Our approach covers (i) a threedimensional description of the quantum dot device, (ii) numerically exact treatment of the electron-electron interaction, (iii) a tilted magnetic field, (iv) the ellipticity of the quantum dot, and (v) both the Rashba and the Dresselhaus spin-orbit coupling, the latter with both linear and cubic terms. We have attributed the observed nonlinear behavior of the singlet-triplet energy gap at $B \lesssim 0.5 \mathrm{~T}$ to the ellipticity of the quantum dot. Then we found a good agreement between theory and experiment in the triplet-singlet energy splitting and in the relaxation rate, although the optimal tilting angle of the magnetic field was found to be considerably smaller than the experimental value. Finally we have explicitly shown that the three-dimensional model is essential to obtain a reasonable agreement with the experiment in both the singlet-triplet energy splitting and the spin relaxation rate.

In view of our results with aspects (i) $-(\mathrm{v})$ listed above included, the origins behind the remaining quantitative deviations between experiment and theory-especially regarding the different angle of the magnetic field-is unclear. However, as the most probable scenario we may suggest that the actual confining potential deviates from harmonic, e.g., by being more strongly confined at the edge of the quantum dot. This can be induced by the gate geometry of the lateral device and/or the nonuniformity of the confinement in the vertical $(z)$ direction. These conditions can increase the high sensitivity of the spectrum to the tilted magnetic field (Fig. 1) even further. In this respect, we hope that the present study motivates more efforts in this direction, both theoretically and experimentally.

\section{ACKNOWLEDGMENTS}

We thank L. M. K. Vandersypen and T. Meunier for valuable comments. This work was supported by the Bergen Research Foundation (Norway), the Research Council of Norway (RCN), the Academy of Finland, the Wihuri Foundation, the Nordforsk Network, and NORDITA.
${ }^{1}$ J. R. Petta, A. C. Johnson, J. M. Taylor, E. A. Laird, A. Yacoby, M. D. Lukin, C. M. Marcus, M. P. Hanson, and A. C. Gossard, Science 309, 2180 (2005).

${ }^{2}$ R. Hanson, L. P. Kouwenhoven, J. R. Petta, S. Tarucha, and L. M. K. Vandersypen, Rev. Mod. Phys. 79, 1217 (2007).

${ }^{3}$ T. Fujisawa, T. H. Oosterkamp, W. G. van der Wiel, B. W. Broer, R. Aguado, S. Tarucha, and L. P. Kouwenhoven, Science 282, 932 (1998).

${ }^{4}$ H. Meijer and D. Polder, Physica 19, 255 (1953).

${ }^{5}$ J. D. Zook, Phys. Rev. 136, A869 (1964).

${ }^{6}$ J. P. Leburton, J. Pascual, and C. M. Sotomayor-Torres, Phonons in Semiconductor Nanostructures (Kluwer Academic Publishers, Dordrecht, 1993).

${ }^{7}$ V. N. Golovach, A. Khaetskii, and D. Loss, Phys. Rev. B 77, 045328 (2008).

${ }^{8}$ J. I. Climente, A. Bertoni, G. Goldoni, M. Rontani, and E. Molinari, Phys. Rev. B 76, 085305 (2007).

${ }^{9}$ V. N. Golovach, A. Khaetskii, and D. Loss, Phys. Rev. Lett. 93, 016601 (2004).

${ }^{10}$ J. I. Climente, A. Bertoni, G. Goldoni, M. Rontani, and E. Molinari, Phys. Rev. B 75, 081303(R) (2007).

${ }^{11}$ A. V. Khaetskii and Y. V. Nazarov, Phys. Rev. B 64, 125316 (2001).
${ }^{12}$ M. Florescu and P. Hawrylak, Phys. Rev. B 73, 045304 (2006).

${ }^{13}$ D. Chaney and P. A. Maksym, Phys. Rev. B 75, 035323 (2007).

${ }^{14}$ S. Dickmann and P. Hawrylak, J. Supercond. 16, 387 (2003).

${ }^{15}$ T. Meunier, I. T. Vink, L. H. Willems van Beveren, K.-J. Tielrooij, R. Hanson, F. H. L. Koppens, H. P. Tranitz, W. Wegscheider, L. P. Kouwenhoven, and L. M. K. Vandersypen, Phys. Rev. Lett. 98, 126601 (2007)

${ }^{16}$ R. Hanson, L. H. Willems van Beveren, I. T. Vink, J. M. Elzerman, W. J. M. Naber, F. H. L. Koppens, L. P. Kouwenhoven, and L. M. K. Vandersypen, Phys. Rev. Lett. 94, 196802 (2005).

${ }^{17}$ T. Fujisawa, D. G. Austing, Y. Tokura, Y. Hirayama, and S. Tarucha, Nature (London) 419, 278 (2002).

${ }^{18}$ J. M. Elzerman, R. Hanson, L. H. Willems van Beveren, B. Witkamp, L. M. K. Vandersypen, and L. P. Kouwenhoven, Nature (London) 430, 431 (2004).

${ }^{19}$ M. Kroutvar, Y. Ducommun, D. Heiss, M. Bichler, D. Schuh, G. Abstreiter, and J. J. Finley, Nature (London) 432, 81 (2004).

${ }^{20}$ A. C. Johnson, J. R. Petta, J. M. Taylor, A. Yacoby, M. D. Lukin, C. M. Marcus, M. P. Hanson, and A. C. Gossard, Nature (London) 435, 925 (2005).

${ }^{21}$ D. M. Zumbühl, J. B. Miller, C. M. Marcus, K. Campman, and A. C. Gossard, Phys. Rev. Lett. 89, 276803 (2002). 
${ }^{22}$ S. M. Reimann and M. Manninen, Rev. Mod. Phys. 74, 1283 (2002).

${ }^{23}$ L. P. Kouwenhoven, D. G. Austing, and S. Tarucha, Rep. Prog. Phys. 64, 701 (2001).

${ }^{24}$ E. Räsänen, J. Könemann, R. J. Haug, M. J. Puska, and R. M. Nieminen, Phys. Rev. B 70, 115308 (2004).

${ }^{25}$ E. Räsänen, H. Saarikoski, A. Harju, M. Ciorga, and A. S. Sachrajda, Phys. Rev. B 77, 041302(R) (2008).

${ }^{26}$ M. C. Rogge, E. Räsänen, and R. J. Haug, Phys. Rev. Lett. 105, 046802 (2010).

${ }^{27}$ P. Stano and J. Fabian, Phys. Rev. B 72, 155410 (2005).

${ }^{28}$ J. J. Krich and B. I. Halperin, Phys. Rev. Lett. 98, 226802 (2007).

${ }^{29}$ D. M. Zumbühl, J. B. Miller, C. M. Marcus, D. Goldhaber-Gordon, J. S. Harris, K. Campman, and A. C. Gossard, Phys. Rev. B 72, 081305 (2005).
${ }^{30}$ V. Popsueva, R. Nepstad, T. Birkeland, M. Førre, J. P. Hansen, E. Lindroth, and E. Walterson, Phys. Rev. B 76, 035303 (2007).

${ }^{31}$ A. Harju, S. Siljamäki, and R. M. Nieminen, Phys. Rev. Lett. 88, 226804 (2002).

${ }^{32}$ U. Bockelmann, Phys. Rev. B 50, 17271 (1994).

${ }^{33}$ J. I. Climente, A. Bertoni, G. Goldoni, and E. Molinari, Phys. Rev. B 74, 035313 (2006).

${ }^{34}$ B. K. Ridley, Quantum Processes in Semiconductors (Clarendon Press, Oxford, 1982).

${ }^{35}$ S. A. Sørngård, M. Førre, and J. P. Hansen, New J. Phys. 14, 013035 (2012).

${ }^{36}$ J. I. Climente, A. Bertoni, G. Goldoni, and E. Molinari, Phys. Rev. B 75, 245330 (2007).

${ }^{37}$ V. I. Fal'ko, B. L. Altshuler, and O. Tsyplyatyev, Phys. Rev. Lett. 95, 076603 (2005). 